\title{
Importancia de la contrainteligencia \\ en la corrupción ${ }^{1}$
}

MARTha ARENAS MolinA, ${ }^{2, *}$

Resumen

El Estado colombiano ha luchado durante muchas décadas contra el flagelo de la corrupción. No obstante, durante los últimos años, este problema se ha agravado y ha traspasado su concepción tradicional -el soborno, la adjudicación indebida de contratos, el clientelismo y el desvío de recursos públicos- para convertirse en un proceso más amplio en el cual se encuentran vinculados actores y acciones procedentes del narcotráfico y de grupos al margen de la ley.

Estas prácticas corruptas, procedentes de insurgentes infiltrados en las Fuerzas Militares, narcotraficantes y personal activo de la fuerza pública al servicio de grupos al margen de la ley, impiden materializar los principios y los objetivos del Estado Social de Derecho y de sus instituciones, en la medida en que concentran en manos de unos pocos los recursos y bienes que deberían destinarse a intereses colectivos; aumentan los costos de administración de bienes y servicios públicos; centran la toma de decisiones en favor de intereses particulares; erosionan la confianza ciudadana en las instituciones y el respecto por la autoridad, y contribuyen a debilitar la legitimidad del Estado colombiano.

Es por ello que, con la implementación de las labores de contrainteligencia, se puede lograr una reducción
${ }^{1}$ Artículo de investigación.

${ }^{2}$ Magíster en Educación de la Pontificia Universidad Javeriana. ‘arenas_martha@yahoo.com.

Fecha de recepción 17 de febrero de 2017

Fecha de aceptación: 12 de agosto de 2017

Para citar este artículo: Arenas, M. (2018). Importancia de la contrainteligencia en la corrupción. Perspectivas en inteligencia, 10(19): 71-83. 
significativa de la corrupción al interior de las Fuerzas Militares. De igual forma se logrará mitigar el número de personal infiltrado en la institución y del personal al servicio de los grupos narcoterrorista.

Palabras claves: corrupción, Fuerzas Militares, contrainteligencia, inteligencia, Colombia.

Clasificación JEL: K11, H56, L85, N46.

\section{Abstract}

The colombian state has struggled for decades against the scourge of corruption, however in recent years this problem has worsened and has transferred its traditional conception (bribery, improper award of contracts, patronage and the diversion of resources public) to become a broader process in which actors and actions are linked from drug trafficking and groups outside the law.

These corrupt practices from these actors, prevent materialize the principles and objectives of the rule of law and its institutions, to the extent that concentrate in the hands of a few resources and assets that should be allocated to collective interests, increase administration costs of goods and public and private services, distort decisions in favor of special interests undermine the respect for authority, erode public trust in institutions, and contribute to undermine the legitimacy of the Colombian state.

That is the reason for the implementation of counterintelligence labor: achieve a significant reduction of corruption within the Armed Forces. At same time, this achieve will accomplish a significant reduction in the number of staff infiltrated the institution and the staff working for the narco-terrorist groups.

Keywords: corruption, military, counterintelligence, intelligence, Colombia.

JEL classification: K11, H56, L85, N46. 


\section{Introducción}

La corrupción es considerada como uno de los fenómenos globales que afecta la institucionalidad, el desempeño, la legitimidad y la credibilidad de las instituciones públicas de todos los países, bajo cualquier sistema de gobierno, generando efectos devastadores a nivel social, económico y político. En este sentido, el Estado colombiano no ha sido ajeno a esta problemática y, por el contrario, tanto las instituciones públicas como privadas han sido permeadas por este problema.

Colombia, al igual que los países industrializados, ha sufrido los flagelos que genera la corrupción, pero a diferencia de otros Estados, el contexto problemático -conflicto armado interno, narcotráfico, consecución de intereses privados- en el que se desarrollan las instituciones tanto públicas como privadas, ha agravado la situación, impidiendo la creación de estrategias eficaces para combatir esta problemática.

Las diferentes mediciones realizadas sobre la corrupción en Colombia muestran un panorama bastante desalentador. Transparencia por Colombia (2011) determinó, según sus mediciones internacionales, que Colombia obtuvo un puntaje de 3,5 -siendo "0" la calificación más baja, es decir alta percepción de corrupción, y 10 la más alta-, es decir, la percepción de corrupción más baja. "Esto sitúa al país en el puesto 78 entre 178 países evaluados. Así mismo, en el contexto americano, Colombia ocupa el décimo puesto entre 18, y en el latinoamericano, el cuarto más alto" (p. 1).

En este sentido, vale la pena aclarar que la corrupción se ha incrementado en los últimos años debido a que el país ha librado una larga lucha contra el narcotráfico y contra los grupos al margen de la ley, lo cual ha traído como consecuencia que los grandes capos del narcotráfico, los cabecillas de grupos narcoterroristas y los líderes de las bandas criminales se hayan fijado como meta la infiltración de las instituciones políticas y militares -Ejército Nacional, Fuerza Aérea Colombiana, Armada Nacional- para poder obtener información que les permita llevar a cabalidad sus planes criminales. Para lograr la obtención de sus objetivos, estos grupos narcoterroristas utilizan estrategias corruptas como el soborno, la amenaza, la presión política y la infiltración en las diferentes organizaciones, con lo cual obtienen información importante que les permite dar golpes contundentes a la fuerza pública y a la población civil.

Teniendo en cuenta que las Fuerzas Armadas Revolucionarias de Colombia (FARC), el Ejército Nacional de Liberación Nacional (ELN) y las Autodefensas 
Unidas de Colombia (AUC) son grupos al margen de la ley que buscan conseguir sus intereses a través del ejercicio de presión contra el gobierno nacional, mediante atentados contra la población civil y la fuerza pública, es necesario que el Estado respondan a estos ataques por intermedio de las Fuerzas Armadas colombianas, con la implementación de medidas de seguridad al interior de sus instituciones que permitan contrarrestar la fuga de información e impidan la infiltración de personal de grupos narcoterroristas.

En este sentido, es necesario enfatizar que los grupos narcoterroristas han decidido justificar dichas infiltraciones en las Fuerzas Militares -dentro de su paradigma marxista leninista-, como un mecanismo de ataque y perjuicio contra las instituciones y agencias encargadas de la seguridad nacional del país.

Para conseguir este fin, estos grupos terroristas entrenan a jóvenes y adultos para que evadan los diversos filtros y logren incorporarse estratégicamente en las filas de la fuerza pública o de las agencias estatales, logrando la obtención de cooperación de los agentes pertenecientes a cada una de las instituciones con la finalidad de "filtrar la información y revelar las operaciones secretas que adelantan en su contra los organismos de seguridad del Estado" (Palacios, 2003, 15).

Ahora bien, no solamente los grupos narcoterrorista constituyen una amenaza a la seguridad interna de la fuerza pública y de las entidades del Estado, sino que las organizaciones delincuenciales también están vinculadas. De esta forma, es necesario examinar el papel que ha jugado la contrainteligencia como ente encargado de garantizar la seguridad interna de las instituciones, al neutralizar conductas delictivas procedentes de grupos al margen de la ley, de bandas de crimen organizado y de personal de las Fuerzas Militares que colabora con estos grupos con la filtración de información. De igual manera, se hace necesario entender el rol que ha jugado la contrainteligencia para combatir y neutralizar la corrupción al interior de las instituciones militares, cuáles han sido las ventajas y logros que ha proporcionado la contrainteligencia para llevar a término las operaciones y estrategias implementadas por el Ejército Nacional, así como los aciertos que ha tenido en la detención de personal infiltrado dentro de las fuerzas.

De otro lado, se debe entender que la corrupción no es solamente un problema de cada una de las instituciones que lo padece, sino que, por el contrario, este es un problema de Estado y es allí en donde se deben afianzar los controles para combatirla. No obstante, se debe reconocer que también es un problema social, por lo cual se hace necesario la creación de un entorno ético que ayude a consolidar una estrategia a largo plazo para combatir este problema (Álvarez, 2007). 


\section{La corrupción en Colombia}

En sociedades como la colombiana -que ha librado problemas como el narcotráfico, el conflicto armado interno y el tráfico de influencias-, es necesario definir el concepto de corrupción y el papel que han jugado actores procedentes del narcotráfico y de grupos al margen de la ley, para agudizar el problema. Tradicionalmente, la corrupción ha sido definida por Transparencia Internacional (2009) como "el abuso de posiciones de poder o de confianza, para el beneficio particular en detrimento del interés colectivo realizado a través de ofrecer o solicitar, entregar o recibir bienes o dinero en especie, en servicios o beneficios, a cambio de acciones, decisiones y omisiones" (p. 1).

Así, el concepto de corrupción incluye tres elementos: “1) El mal uso del poder, 2) Un poder encomendado, es decir, puede estar en el sector público o privado, 3) Un beneficio privado". (Carbou, 2012, 2). Sin embargo, esta definición se queda corta para entender la complejidad del fenómeno en sociedades como la colombiana. En este sentido, es necesario abordar la corrupción como:

... un conjunto de modalidades delictivas que se lleva a cabo en las instituciones, públicas y privadas, tanto a nivel político, económico, militar, o social, a través del cual se busca la satisfacción de intereses de grupos poderosos legales o ilegales, en detrimento de intereses y propósitos de la colectividad, violando la ley y menoscabando lo derechos de los semejantes. (Ungar, 2010, 1).

\subsection{Características y tipificación de la corrupción en Colombia}

El ejercicio de la corrupción presenta varias facetas que van desde la desviación de recursos públicos hacia arcas particulares, pasando por el soborno de empresas particulares a servidores públicos, hasta llegar al extremo de hacer desaparecer a quienes se le oponen, bien sean políticos, o simplemente ciudadanos que reclaman sus derechos. (Interpol, s.f., 2)

La ley penal colombiana, por ejemplo, sanciona dieciocho modalidades diferentes de la corrupción en el sector público que abarcan entre otros:

- El abuso del cargo público.

- La expedición de actos arbitrarios.

- La intervención en las tramitaciones.

- Los intereses en beneficio de terceros.

- Los dictámenes contrarios a la ley.

- La omisión o retardo de actos públicos.

- La asesoría ilícita, la omisión de denuncias. 
- La revelación indebida de documentos.

- La utilización en provecho propio de información reservada.

- La representación o asesoría ilegales. (Flores et al., s.f.)

\section{La contrainteligencia en las Fuerzas Militares}

Se entiende como "la parte de la inteligencia encargada de neutralizar y controlar la agresión enemiga, real o potencial. La agresión se puede expresar a través de acciones de espionaje contra información y documentos, atentados contra el personal y las instalaciones, sabotaje contra equipos y demás elementos técnicos y electrónico" (Valbuena, 2003, 10). Esta actividad es realizada por personal especializado reunido en la sección o subsección de contrainteligencia. Adicionalmente estas secciones tienen la función de asesorar al comando para que lleve a cabo las respectivas investigaciones que garanticen la seguridad interna de las Fuerzas Militares.

\subsection{Tipos de contra inteligencia}

Contrainteligencia táctica: "Son las medidas y operaciones de contrainteligencia realizadas en apoyo directo al personal operativo", las cuales comprenden (Valbuena, 2003, 10):

- Seguridad en las operaciones.

- Estudio de Seguridad al Personal (ESP).

- Estudios de lealtad.

- Entrevistas.

- Redes de contrainteligencia.

- Estudios de seguridad a las instalaciones.

Contrainteligencia estratégica: "Son las medidas y operaciones de contrainteligencia hechas a nivel nacional contra las amenazas internas y externas del estado" (Valbuena, 2003, 10).

\subsection{Modalidades de contrainteligencia}

Pasivas: "Aquellas que se adoptan para evitar que el enemigo tenga acceso a la información, se consideran defensivas y se emplean en la seguridad de instalaciones, documentos, material clasificado, disciplina del secreto y compartimentación" (Palacios, 2003, 10).

Activas: "Aquellas que destruyen o neutralizan los elementos de inteligencia enemigos. Se orientan a descubrir las agencias y métodos para evitar su acción 
o desorientarla. Dentro de esta modalidad se encuentran los estudios de seguridad personal y la entrevista de confidencialidad" (Palacios, 2003, 10).

\section{La corrupción al interior de las Fuerzas Militares}

\subsection{Problemática de la corrupción en las Fuerzas Militares}

Históricamente, las instituciones públicas y privadas del Estado colombiano han sufrido una serie de problemas de corrupción. Si bien no se debe desconocer que los mayores índices de corrupción son pertenecientes a las instituciones políticas, al interior de las Fuerzas Militares también se han presentado algunos problemas de corrupción que han impedido la materialización de muchas estrategias y golpes en contra de los grupos narcoterroristas. Por lo tanto, es de vital importancia conocer cuáles son las falencias que existen en su interior con el fin de contrarrestar los intentos de estos grupos por infiltrarse en las instituciones y conseguir información para debilitar la seguridad nacional.

Dentro de las falencias más importantes podemos destacar la existencia de una fuga de información sistematizada, por parte del personal infiltrado, ya que en algunas ocasiones no se posee el suficiente control con los equipos de computación y la información contenida en ellos, lo cual podría generar que, con el transcurso del tiempo, las instituciones militares se vean más expuestas a la infiltración por parte de grupos delincuenciales -Bacrim, FARC, ELN y grupos narcoterroristas-, lo que implicaría producir bajas de personal, ser objeto de acciones de sabotaje y la pérdida continua de información obtenida de grupos narcoterroristas. En esa medida, las acciones terroristas podrían llegar a amenazar el orden público y las operaciones de las Fuerzas Militares, ya que con la filtración de información confidencial a grupos al margen de la ley pueden fracasar golpes certeros contra estos grupos ilegales.

Por último, cabe destacar el mal manejo de dineros destinados por parte del Estado para el sostenimiento de la fuerza pública, con la celebración indebida de contratos o compra de equipos tecnológicos, lo cual destruye la eficiencia del Estado, disminuyendo sus recursos y debilitando sus instituciones.

Lo anterior se ve evidenciado durante los grandes golpes que proporcionaron los grupos narcoterroristas al Ejército Nacional durante el año 2007, en el cual se registran la mayor cantidad de infiltraciones en las instituciones castrenses. El primer episodio quedó al descubierto a mediados de julio de 2007, cuando se descubrió que Diego León Montoya, alias "Don Diego", jefe del cartel del 
norte del Valle y uno de los narcotraficantes más buscados del mundo, tenía a su servicio a varios oficiales y suboficiales activos del Ejército.

Como resultado de las investigaciones adelantadas por la Fiscalía, fueron capturados una funcionaria del Comando del Ejército, Ana Carolina Laverde, secretaria del área de hojas de vida del Ejército y siete uniformados, entre oficiales y suboficiales (Caracol, 2009, 1). Así mismo, cuando el país apenas estaba asimilando el escándalo de los militares que trabajaban para "Don Diego", en esa misma época se desarrolló un operativo en un batallón contraguerrilla que dejó al descubierto que las Farc tenían en su poder información estratégica de las Fuerzas Militares.

El 15 de julio, después de un combate con guerrilleros del Frente 42, los militares encontraron en el cuerpo de un subversivo tres discos duros portátiles y varias memorias USB de alta capacidad. Cuando los analistas comenzaron a mirar el contenido, encontraron datos importantes sobre información militar clasificada. Nombres de los oficiales que participaban en las operaciones, listas de la red de cooperantes y hasta mapas de algunas operaciones militares estaban en poder de la guerrilla (La Nación, 2007, 1).

Con estos casos quedó evidenciado que era necesario fortalecer los controles al interior de las Fuerzas Militares para evitar que casos como estos se repitieran y se debilitara la confianza de la población en las instituciones públicas, encargadas de garantizar la seguridad nacional.

\subsection{Consecuencias de la corrupción para las Fuerzas Militares}

La corrupción al interior de las Fuerzas Militares de un Estado es un problema que no solamente afecta el interior de la institución, sino que además afecta la seguridad y estabilidad de todo un país, al colocar en riesgo el orden público y las operaciones de esas entidades ya que, al generarse una fuga de información confidencial, permite a grupos al margen de la ley evitar los controles de las autoridades y evadir los golpes que se pretenden dar en contra de sus estructuras delincuenciales.

No obstante, este no es el único problema que trae la corrupción al interior de las instituciones militares, ya que, al igual que en otras instituciones estatales, existe un mal manejo de recursos por parte de algunos funcionarios, ya sea a través de la compra de equipos innecesarios o del uso inadecuado de recursos destinados por el Estado, lo cual disminuye notablemente la eficiencia de las fuerzas militares y, por ende, la consecución de sus objetivos.

Esto queda plasmado a través de las distintas investigaciones llevadas a cabo en la Comisión Segunda de la Cámara de Representantes, ante la cual el senador 
Camilo Romero reveló una carta para evidenciar la inversión de recursos del Estado en la construcción de los kioscos usados de manera irregular por militares detenidos dentro de la cárcel de Tolemaida.

Según esta carta, el primero de los kioscos fue construido en 1998 y el último en enero de 2010, con una donación de la Sexta Brigada del Ejército. Así mismo, ante la Comisión Segunda del Senado, el General Alejandro Navas admitió su responsabilidad por las irregularidades en la cárcel de Tolemaida entre el 6 de agosto de 2010 y el 17 de enero de 2011, admitiendo que en el centro de reclusión se comprobaron irregularidades relacionadas con ausencia de control, falta de actas de entradas y salidas y entrada de elementos prohibidos (Semana, 2011, 1).

Otro de los graves problemas de corrupción que afrontan las fuerzas militares está relacionado con el tráfico de influencias en su interior, lo cual genera que se tomen decisiones para privilegiar las actuaciones y actividades de grupos al margen de la ley, ya sea para permitir que se trafique con armas y drogas o para dar paso a los insurgentes por las diferentes regiones del país.

Uno de los ejemplos más destacados fue el caso del suboficial Manuel Antonio Calderón Barragán, de la dotación del Comando del Ejército, el cual fue detenido por tener nexos con los jefes del Frente 43 de las Fuerzas Armadas Revolucionarias de Colombia (FARC). El Sargento Calderón Barragán, de 29 años, y quien era uno de los quince hombres más cercanos al jefe de ese frente, Géner García Molina alias “Jhon 40”, fue capturado por agentes de inteligencia, junto a otros colaboradores de las FARC (El Siglo de Torreón, 2007).

Así mismo, el Sargento Calderón tenía una oficina en el segundo piso del Comando Central del Ejército de Colombia y sostenía reuniones periódicas con el mencionado jefe de las FARC, quien estaba pedido en extradición por Estados Unidos. Calderón tenía acceso a las hojas de vida del personal del Ejército y, según el proceso en su contra, era el encargado de la compra de granadas, munición y armas cortas y largas para el Frente 43.

De esta manera, se puede afirmar que todos estos casos de corrupción al interior de las instituciones militares producen inestabilidad institucional, pérdida de legitimidad y de credibilidad por parte de la población civil.

\section{Importancia de la contrainteligencia}

La contrainteligencia juega un papel fundamental para garantizar la seguridad de las instituciones militares y de las entidades del Estado, ya que tiene como 
finalidad negar la información propia al enemigo. Con ello se contribuye a proporcionar libertad de acción al comandante, evitando que el enemigo conozca tanto sus intenciones como lo que se sabe de él. De otro lado, ayuda a impedir que el enemigo obtenga información sobre vulnerabilidades y formas de acción de las fuerzas propias; el conocimiento propio de su situación y el éxito logrado por sus actividades de Inteligencia (Manual de contrainteligencia, s.f.)

Adicionalmente, por medio delas investigaciones llevadas a cabo por las agencias de contrainteligencia a nivel local nacional y departamental, se pueden detectar los planes de infiltración y penetración de las organizaciones terroristas, especialmente FARC, ELN, y organizaciones dedicadas al narcotráfico.

Con esto se contribuye a "mejorar la seguridad de las bases militares, de los archivos confidenciales, de las bases informáticas y archivos electrónicos, resguardando la información privilegiada de las fuerzas militares, la cual es vital para dar golpes certeros a estos grupos narcoterroristas" (Palacios, 2003, 16).

Por otra parte, es posible detectar comportamientos irregulares por parte de miembros de la institución que estén al servicio de grupos terroristas, ya sea mediante el traspaso de información o como facilitadores para el ingreso de personal infiltrado en la institución, con el objetivo de conocer más de cerca las actividades y misiones desempeñadas por los miembros de las Fuerzas Militares y de los altos mandos.

Así mismo con las tácticas de contrainteligencia se pueden identificar los problemas de la región tanto en el aspecto político, social, y económico, analizando el área gubernamental, partidos políticos, campo social (salud, educación, recreación, servicios públicos, costumbres, problemas regionales entre otros), campo económico, medios de comunicación, transporte y vías de comunicación; todo esto con el fin de implementar el trabajo a cubierto, así como las historias ficticias de cada uno de los integrantes (Beltrán, 2003, 11).

Por último, se logra que los dineros destinados para la compra de tecnología, mejoramiento de instalaciones, adquisición de armamento, entre otras, sea utilizada de manera óptima, mejorando la eficiencia en la utilización de los recursos y en el funcionamiento de las instituciones militares.

\section{Estudio de caso sobre la infiltración y penetración del ELN en San Vicente de Chucuri (Santander)}

Para poder identificar y comprender cuál es el papel que ha jugado la contrainteligencia al interior de las Fuerzas Militares de Colombia, es necesario 
entender el contexto en el cual se ha librado la guerra contra los grupos narcoterroristas. Así mismo, establecer cuáles son las tácticas más utilizadas por estos grupos para poder infiltrarse al interior de las instituciones militares y lograr sus objetivos.

Es por ello que mediante el análisis de un estudio de caso se pueden detectar las estrategias implementadas por estos grupos y las falencias existentes al interior de las Fuerzas Militares colombianas, con el objetivo de mejorar las fallas e implementar de manera adecuada las medidas necesarias para garantizar la seguridad nacional.

El antecedente más significativo en la organización narcoterrorista ELN con respecto al plan de infiltración y penetración se registra con los hechos ocurridos en el Batallón de Infantería Luciano D’Luyer, en San Vicente Chucurí (Santander). A las instalaciones del batallón se presenta un subversivo del ELN, integrante del frente "Capitán Parmenio", perteneciente al frente de guerra nororiental, quien se identifica como Jesús Uribe. Este sujeto suministra algunas informaciones al Ejército a través de las cuales se logra dar golpes a la estructura delincuencial, ganándose la confianza del comandante del batallón quien, sin una plena verificación de antecedentes y comprobación de su lealtad, termina convirtiéndose en el escolta personal y hombre de confianza del comandante.

El insurgente logra tener acceso a la información que la sección segunda de la unidad poseía sobre el orden de batalla de los diferentes grupos subversivos en la jurisdicción. No obstante, la entrega de otro insurgente con información más actualizada hizo que la información suministrada por Jesús Uribe pasara a un segundo plano y no fuera de gran relevancia para el Ejército Nacional, razón por la cual el comandante de la unidad decide que la situación de Jesús Uribe debe definirse, ya que no podía permanecer más tiempo en la unidad.

Este comentario es escuchado por un soldado que trabajaba en el comando del batallón el cual, por congratularse con Jesús Uribe, decide contarle lo sucedido. Esto ocasiona que el subversivo decida terminar con su misión y su oportunidad se presenta durante el desarrollo del paro nororiental en el sector de la Llana Santander, en el cual se origina un enfrentamiento a bala entre manifestantes y miembros del Ejército, siendo asesinado el comandante del batallón por la espalda, por el insurgente Jesús Uribe.

En esta posición privilegiada el insurgente cumplía la misión de obtener información importante y rendir informes periódicos a los cabecillas de la organización sobre aspectos relacionados con operaciones militares, seguridad 
del batallón D’Luyer y las bases de patrullaje, información que la unidad poseía sobre los bandidos (Valbuena, 2003).

De esta manera queda evidenciado que, en algunas ocasiones, miembros pertenecientes a las Fuerzas Militares descuidan algunas medidas de seguridad (estudios de seguridad, rectificación de antecedentes, entrevistas de confidencialidad), lo cual permite a los insurgentes infiltrarse en las estructuras, filtrar información confidencial y entorpecer la labor de las Fuerzas Militares.

\section{Conclusiones}

La corrupción es un flagelo que ha golpeado de manera directa el funcionamiento de las instituciones estatales en todas sus áreas. No obstante, la contrainteligencia ha surgido como una estrategia eficiente y eficaz que ha permitido la consecución de los fines esenciales del Estado, la vigencia del régimen democrático y la seguridad y defensa de la Nación, previniendo las amenazas contra las personas residentes en Colombia y los ciudadanos colombianos en todo tiempo y lugar.

La contrainteligencia ha permitido la implementación de medidas tendientes a garantizar la protección de la población y las instituciones democráticas frente a las amenazas como el terrorismo, el narcotráfico, el secuestro, el tráfico de armas, municiones, explosivos y otros materiales relacionados, el lavado de activos y otras amenazas de igual naturaleza.

La contrainteligencia también ha jugado un papel fundamental para prevenir, neutralizar, combatir y destruir de manera efectiva y certera las acciones enemigas tendientes a la destrucción o captación de información privilegiada. No obstante, es necesario que se continúe avanzando en la implementación de medidas de seguridad al interior de las Fuerzas Militares, las cuales permitan contrarrestar las nuevas estrategias implementadas por los grupos narcoterroristas.

De igual manera, la contrainteligencia debe ser dinámica y responder a las fluctuaciones y constantes cambios del mundo globalizado, teniendo en cuenta que con el trascurso de los años emergen nuevos riesgos y amenazas que deben ser contrarrestados de manera eficaz para garantizar la seguridad nacional.

Finalmente, la Contrainteligencia debe enfatizar todas sus estrategias en el carácter anticipativo y preventivo, previniendo las posibles amenazas procedentes de los grupos armados ilegales que pongan en riesgo la estabilidad de la democracia y de la población civil. 


\section{Referencias}

1. Álvarez, E. (2007). La lucha contra la corrupción en Colombia como herramienta para un control social exacerbado. Recuperado el 18 de junio de 2012 de http://aprendeenlinea. udea.edu.co/revistas/index.php/red/art.

2. Beltrán, A., y Salcedo, B. (2003). Red de contrainteligencia interna y externa. Escuela de Inteligencia y Contrainteligencia. BG. Ricardo Charry Solano.

3. Caracol (2009). Aseguran dos presuntos infiltrados de las Farc en el Ejército. Recuperado el 18 de junio de 2012 de http://www.caracol.com.co/nota.aspx?id=776935

4. Carbou, J. (2012). El estado supremo de la corrupción es constitucional. Recuperado el 19 de junio de 2012 de http://www.revistaperspectiva.com/detalle.php?Revista=23\&Art...

5. El siglo de torreón (2007). Capturan a un militar infiltrado por las FARC en el Ejército colombiano. Recuperado el 19 de junio de 2012 de www.elsiglodetorreon.com. $\mathrm{mx} /$.../318715.capturan-a-un-militar-infilt...

6. Flórez, A., Ospina, J., Pava, J., Gómez, J., Venegas, J., Salazar, G., Lozano, M., Prada, O., Franco, R., Sánchez, W. (s.f.). Estrategias contra la corrupción. Recuperado el 18 de junio de 2012 de http://www.interamericanusa.com/

7. Interpol (S.f.). Recursos policiales -lucha contra la corrupción. Recuperado el 22 de junio de 2012 de http://www.interpol.int/es/Conocimientos/Formación-y-perfecc...

8. La Nación. (2007). Las FARC y los narcos, infiltrados en el ejército. Recuperado el 22 de junio 2012 de http://www.lanacion.com.ar/930302

9. Manual de contrainteligencia. (s.f). La Contrainteligencia. Capitulo Vll. Recuperado el 22 de junio de 2012 de www.ese.mil.ve/manuales/Manual\%20de\%20Inteligencia/9Mi-c-7. pdf

10. Palacios, A. (2003). El papel de la contrainteligencia en la seguridad informática. Escuela de Inteligencia y Contrainteligencia. BG. Ricardo Charry Solano

11. Semana. (2001). Tolemaida resort. Recuperado el 23 de junio de 2012 de http:// www. semana.com/nacion/tolemaida-resort/154427-3.aspx

12. Transparencia por Colombia (2011). Índice de Transparencia Nacional 2008-2009, radiografía de riesgos de corrupción en el Estado colombiano y sus empresas. Recuperado el 23 de junio de 2012 de http:// www.transparenciacolombia.org.co/LinkClick. aspx?link=101...62.

13. Transparencia Internacional (2009). Informe Global de la Corrupción 2009: Corrupción y sector privado. Recuperado de: https://www.transparency.org/whatwedo/publication/ informe_global_de_la_corrupcion_2009_corrupcion_y_sector_privado

14. Ungar, E. (2010). La corrupción en Colombia, cáncer de la democracia. Recuperado el 24 de junio de 2012 de http// razonpublica.com/index...y.../1510-la-corrupcion-en-colombia. html

15. Valbuena, B. (2003). Las entrevistas de confiabilidad en las labores de contrainteligencia. Escuela de Inteligencia y Contrainteligencia. BG. Ricardo Charry Solano 